\title{
On the chlorophyll $a$ fluorescence yield in chloroplasts upon excitation with twin turnover flashes (TTF) and high frequency flash trains
}

\author{
Wim Vredenberg · Milan Durchan · Ondrej Prasil
}

Received: 24 August 2006/Accepted: 19 February 2007/Published online: 8 May 2007

(C) Springer Science+Business Media B.V. 2007

\begin{abstract}
Chlorophyll fluorescence is routinely taken as a quantifiable measure of the redox state of the primary quinone acceptor $\mathrm{Q}_{\mathrm{A}}$ of PSII. The variable fluorescence in thylakoids increases in a single turnover flash (STF) from its low dark level $F_{\mathrm{o}}$ towards a maximum $F_{\mathrm{m}}^{\mathrm{STF}}$ when $\mathrm{Q}_{\mathrm{A}}$ becomes reduced. We found, using twin single turnover flashes (TTFs) that the fluorescence increase induced by the first twin-partner is followed by a $20-30 \%$ increase when the second partner is applied within $20-100 \mu$ s after the first one. The amplitude of the twin response shows a period-of-four oscillation associated with the 4-step oxidation of water in the Kok cycle (S states) and originates from two different trapped states with a life time of $0.2-0.4$ and $2-5 \mathrm{~ms}$, respectively. The oscillation is supplemented with a binary oscillation associated with the two-electron gate mechanism at the PSII acceptor side. The $F(t)$ response in high frequency flash trains $(1-4 \mathrm{kHz})$ shows (i) in the first 3-4 flashes a transient overshoot $20-30 \%$ above the $F_{\mathrm{m}}^{\mathrm{STF}}=3^{*} F_{\mathrm{o}}$ level reached in the 1 st flash with a partial decline towards a dip D in the next 2-3 ms, independent of the flash frequency, and (ii) a frequency independent rise to
\end{abstract}

W. Vredenberg $(\square)$

Laboratory of Plant Physiology, Wageningen University and Research, Arboretumlaan 4, 6703 BD Wageningen,

The Netherlands

e-mail: wim.vredenberg@wur.nl

M. Durchan · O. Prasil

Faculty of Biology and Institute of Physical Biology,

University of South Bohemia, Ceske Budejovice, Czech

Republic

O. Prasil

Laboratory of Photosynthesis, Institute of Microbiology,

Academy of Sciences Czech Republic, Trebon, Czech Republic
$F_{\mathrm{m}}=5^{*} F_{\mathrm{o}}$ in the $3-60 \mathrm{~ms}$ time range. The initial overshoot is interpreted to be due to electron trapping in the $S_{0}$ fraction with $\mathrm{Q}_{\mathrm{B}}$-nonreducing centers and the dip to the subsequent recovery accompanying the reoxidation of the double reduced acceptor pair in these RCs after trapping. The rise after the overshoot is, in agreement with earlier findings, interpreted to indicate a photo-electrochemical control of the chlorophyll fluorescence yield of PSII. It is anticipated that the double exciton and electron trapping property of PSII is advantageous for the plant. It serves to alleviate the depression of electron transport in single reduced $\mathrm{Q}_{\mathrm{B}}$-nonreducing $\mathrm{RCs}$, associated with electrochemically coupled proton transport, by an increased electron trapping efficiency in these centers.

Keywords Chlorophyll $a$. Fluorescence quenching . $\mathrm{Q}_{\mathrm{B}}$-nonreducing center · Single turnover excitation .

Twin turnover excitation - Three state trapping mechanism (TSTM)

\begin{tabular}{|c|c|}
\hline \multicolumn{2}{|c|}{ Abbreviations } \\
\hline$\beta$ & $\begin{array}{l}\text { Fraction double reduced } \mathrm{Q}_{\mathrm{B}} \text {-nonreducing } \\
\mathrm{RCs}\end{array}$ \\
\hline DCMU & 3(3,4-dichlorophenyl)-1,1-dimethylurea \\
\hline FCCP & $\begin{array}{l}\text { Carbonyl cyanide } p \text { - } \\
\text { trifluoromethoxyphenylhydrazone }\end{array}$ \\
\hline$F(t)$ & Fluorescence level at time $t$ \\
\hline$F_{\mathrm{m}}^{\mathrm{S}(\mathrm{T}) \mathrm{TF}}$ & $\begin{array}{l}\text { Maximum fluorescence induced by STF or } \\
\text { TTF }\end{array}$ \\
\hline$F_{\mathrm{o}}$ & $\begin{array}{l}\text { Intrinsic fluorescence level of system with } \\
100 \% \text { open PSUs }\left(100 \% \mathrm{Q}_{\mathrm{A}}\right)\end{array}$ \\
\hline$F_{\mathrm{v}}$ & Variable fluorescence, $F_{\mathrm{v}}=F_{\mathrm{m}}-F_{\mathrm{o}}$ \\
\hline$\Delta F_{\mathrm{v}}$ & $\begin{array}{l}\text { Increase in variable fluorescence level by } \\
\text { 2nd STF in TTF with } \Delta F_{\mathrm{v}}=F_{\mathrm{m}}^{\mathrm{TTF}}-F_{\mathrm{m}}^{\mathrm{STF}}\end{array}$ \\
\hline
\end{tabular}


$\Delta F^{\mathrm{Qa}} \quad$ Fluorescence increase in STF associated with release of $\mathrm{Q}_{\mathrm{A}}$-quenching

$\Delta F^{\text {Phe }} \quad$ Fluorescence increase in STF associated with release of Phe-quenching

$\Delta F^{\mathrm{nQb}} \quad$ Fluorescence increase in STF associated with release of quenching in $\mathrm{Q}_{\mathrm{B}^{-}}$ nonreducing $\mathrm{RCs}$

$F^{\mathrm{sc}} \quad$ Fluorescence of semi-closed (-open) $\mathrm{Q}_{\mathrm{B}^{-}}$ reducing $\mathrm{RCs}$

$F_{\mathrm{nQb}}^{\mathrm{sc}} \quad$ Fluorescence of semi-closed (-open) $\mathrm{Q}_{\mathrm{B}^{-}}$ nonreducing $\mathrm{RCs}$

$F^{\mathrm{c}}$ nQb $\quad$ Fluorescence of closed $\mathrm{Q}_{\mathrm{B}}$-nonreducing RCs

$k_{-1} \quad$ Rate constant of radical pair recombination

$k_{\mathrm{AB} 1} \quad$ Rate constant of $\mathrm{Q}_{\mathrm{A}}^{-}$oxidation by $\mathrm{Q}_{\mathrm{B}}$

$k_{1,2} \quad$ Rate constant of reoxidation of $\left[\mathrm{PheQ}_{\mathrm{A}}\right]^{2-}$ to

$\left[\mathrm{PheQ}_{\mathrm{A}}\right]^{-}$in $\mathrm{Q}_{\mathrm{B}}$-nonreducing $\mathrm{RCs}$

$k_{\mathrm{nB} 1,2} \quad$ Rate constant of reoxidation $\left[\mathrm{PheQ}_{\mathrm{A}}\right]^{-}$in $\mathrm{Q}_{\mathrm{B}}$-nonreducing $\mathrm{RCs}$

$k_{\mathrm{e}} \quad$ Rate constant of $\mathrm{Q}_{\mathrm{A}}$ photoreduction

MTF Multi turnover light pulse

$N \quad$ Number of STFs in variable flash train

OEC Oxygen evolving complex

$\mathrm{P}_{680} \quad$ Primary electron donor of PSII

Phe Pheophytin, primary electron acceptor of PSII

$\left[\mathrm{PheQ}_{\mathrm{A}}\right]^{2-,-} \quad$ Double and single reduced acceptor pair of PSII, respectively

PQ Plastoquinone

PSII Photosystem II

PSU Photosynthetic unit

$\mathrm{Q}_{\mathrm{A}} \quad$ Primary quinone acceptor of PSII

$\mathrm{Q}_{\mathrm{B}} \quad$ Secondary quinone acceptor of PSII

RC Reaction center of PSII

STF Single turnover flash

$\mathrm{T}_{(\Delta t)} \mathrm{TF} \quad$ Twin turnover flash (with darktime $\Delta t$ (in $\mu \mathrm{s})$ between twin partners)

TSTM Three-state trapping model

$\mathrm{Y}_{\mathrm{Z}} \quad$ Secondary electron donor of PSII

\section{Introduction}

Photosynthesis uniquely performs the transformation of photon (light) energy into several forms of (electro-) chemically convertible energy. It occurs in an integrated set of light- and dark reactions within a highly structured photosynthetic apparatus. The pigments (chlorophylls, carotenoids) of antenna complexes in green cells absorb incoming photons and transfer the excitons to the reaction centers (RCs) where they are photochemically trapped and converted into the energy of separated charges $\mathrm{P}^{+}$and $\mathrm{A}^{-}$of the primary donor $\mathrm{P}$ and acceptor $\mathrm{A}$, respectively. The photosynthetic apparatus is embedded and oriented in the closed and partially folded membrane system (thylakoid) in the chloroplast. The thylakoid membrane anchors the active proton pumps of the two photochemical systems PSI and PSII, the cytochrome $b_{6} f$ complex and of the ATP synthase (Ke 2001).

PSII harbors donor- and acceptor sites where in- and efflux of electrons, respectively is initiated upon excitation. At the PSII donor side electron transfer (donation) from $\mathrm{H}_{2} \mathrm{O}$ occurs in a cascade via the oxygen-evolving complex (OEC) and the secondary donor $\mathrm{Y}_{\mathrm{Z}}$, (a particular Tyr (Tyr161 on the $\mathrm{D} 1$ protein) to the primary donor $\mathrm{P}_{680}^{+}$. At the acceptor side this cascade is from the reduced primary acceptor pheophytin (Phe) via the primary and secondary quinone acceptors $\mathrm{Q}_{\mathrm{A}}$ and $\mathrm{Q}_{\mathrm{B}}$ to the $\mathrm{PQ}$ pool and via the cytochrome $b_{6} f$ complex to PSI (Hankamer et al. 1997). Chlorophyll $a$ (chl) fluorescence, for the major part emitted by PSII antenna chlorophylls, is a de-excitation pathway in the PSUs competitive with photochemical energy trapping (conversion) in RCs resulting in fluorescence quenching when trapping in the RC is effective. The complimentary relation between fluorescence and photochemical yield has made fluorescence monitoring a sensitive non-invasive tool for probing the electron transport in PSII (Papageorgiou and Govindjee 2004). Several models have been presented which quantitatively relate the changes in fluorescence yield to the photochemical yield of electron transport to and from PSII (Bouges-Bocquet 1980; Stirbet et al. 1998; Vredenberg 2000, 2004; Lazár 2003, 2006; Zhu et al. 2005).

The light-dependent chl $a$ fluorescence yield in chloroplasts and intact leaves is variable between a lowest level $F_{\mathrm{o}}$ at full photochemical quenching under darkadapted conditions and a maximal level $F_{\mathrm{m}}$ at saturating light intensities at which quenching is released. Variable fluorescence is defined as $F_{\mathrm{v}}=F_{\mathrm{m}}-F_{\mathrm{o}}$. The primary quinone acceptor $\mathrm{Q}_{\mathrm{A}}$ has become known as the major and principal quencher; the quenching is released upon its photoreduction (Duysens and Sweers 1963). $F_{\mathrm{m}}$ is associated with full reduction of $\mathrm{Q}_{\mathrm{A}}$ and with the trappingincompetent closed RC. Other electron transport intermediates at the acceptor and donor sides of PSII have been proposed as additional functional quenchers like Phe (Phe) (Klimov et al. 1977; Vredenberg 2000), reduced forms of the secondary quinone acceptor $\mathrm{Q}_{\mathrm{B}}^{-}$(Samson et al. 1999; Yaakoubd et al. 2002, Schreiber 2002, 2004) and $\mathrm{Q}_{\mathrm{B}}^{2-}$ (Zhu et al. 2005), plastoquinone (PQ) (Vernotte et al. 1979), oxidized primary $\left(\mathrm{P}_{680}^{+}\right)$(Butler 1972), and secondary donor $\left(\mathrm{Y}_{\mathrm{Z}}^{+}\right)$(Vredenberg et al. 2001) or side products like triplet carotenoids $\left(\mathrm{car}^{\mathrm{T}}\right)$ (Steffen et al. 2001). Their quenching has been discussed in relation to 
the seeming paradox that the release of fluorescence quenching $F_{\mathrm{v}}$ in a single turnover flash (STF) in which all $\mathrm{Q}_{\mathrm{A}}$ is reduced, is substantially below that measured in multi-turnover light pulses (MTF) (Kramer et al. 1995; Samson and Bruce 1996; Kolber et al. 1998; Vasilev and Bruce 1998; Samson et al. 1999; Koblizek et al. 2001; Vredenberg et al. 2005). The documented effect of electric fields on the fluorescence yield (Vos et al. 1991; van Gorkom 1996; Vredenberg 2004) is assumed to be comparatively small in the time domain $<2 \mathrm{~ms}$, and is not considered here.

This paper reports on experiments in which the variable fluorescence (fluorescence induction) in the time range between 0.01 and $10 \mathrm{~ms}$ upon a double (twin) single turnover excitation flash (TTF) has been measured in darkadapted thylakoid membranes. They are aimed to determine the electron trapping efficiency in the second single turnover excitation $\left(\mathrm{STF}_{2}\right)$ of a TTF (twin). This efficiency is dependent on and determined by the lifetime of the fraction of PSII units in which the RC is in an excitable and electron trapping competent state $\left[\mathrm{Y}_{\mathrm{z}} \mathrm{P}_{680} \mathrm{PheQ}_{\mathrm{A}}^{-}\right]$. Formation of this state, which can be identified as temporarily $\mathrm{Q}_{\mathrm{B}}$-nonreducing, is initiated by the 1 st partner $\left(\mathrm{STF}_{1}\right)$ of the TTF (twin) and accompanied by an increase in variable fluorescence $F_{\mathrm{v}}^{\mathrm{STF}} \sim 2^{*} F_{\mathrm{o}}$. Here it is shown that the variable fluorescence in a TTF, when properly tuned in the time window of the lifetime of the RC in state $\left[\mathrm{Y}_{\mathrm{Z}} \mathrm{P}_{680}\right.$ $\left.\mathrm{PheQ}_{\mathrm{A}}^{-}\right]$, can be as high as $F_{\mathrm{v}}^{\mathrm{TTF}} \sim 2.4 * F_{\mathrm{o}}$. The higher TTF response, as compared to that in STF, is ascribed to originate from a fraction of RCs in which double reduction of the acceptor pair $\left[\mathrm{PheQ}_{\mathrm{A}}\right]$ has occurred. This double-hitresponsive fraction is probably identical to the $20-30 \% \mathrm{~S}_{0}$ fraction with $\mathrm{Q}_{\mathrm{B}}$-nonreducing $\mathrm{RCs}$ commonly present in systems that have been dark-adapted for tens of minutes. It is shown, in agreement with earlier results (Vredenberg et al. 2006), that reoxidation of the double reduced acceptor pair in the $S_{0}$ fraction occurs within 2-5 ms. This is 10 -fold slower than reoxidation of the single reduced pair in the complimentary $\left(S_{1}\right)$ fraction.

\section{Materials and methods}

Plant growth (Pea), chloroplast isolations and thylakoid preparation were as described elsewhere (Durchan et al. 2001; Vredenberg et al. 2004). Thylakoid suspending medium ( $\mathrm{pH}$ 7.5) contained (in $\mathrm{mM}$ ) sorbitol, 330; $\mathrm{MgCl}_{2}, 5 ; \mathrm{KCl}, 10 ; \mathrm{MnCl}_{2}, 1$; and Tricine, 50. Room temperature chl fluorescence yields were measured in dark-adapted chloroplast preparations $(1-2 \mu \mathrm{g} / \mathrm{ml})$ with the Dual-Modulation Kinetic Fluorometer (Photon Systems Instruments, Brno, Czech Republic), as described in detail elsewhere (Nedbal et al. 1999). The set-up was routinely used in a mode in which the fluorescence yield during and after $N(60 \geq N \geq 1) 35 \mu$ s single turnover excitations (STFs) in a flash train variable in frequency from 0.0125 to $4 \mathrm{kHz}$ was probed by weak $3.5 \mu$ s measuring flashes, fired at progressing dark intervals in a time domain between $50 \mu \mathrm{s}$ and $18 \mathrm{~s}$ with, on a log time scale 4 equidistant excitations per decade. The response is plotted, usually on a logarithmic time scale, as the fluorescence signal $F(t)$, relative to the dark fluorescence yield $F_{\mathrm{o}} . F_{\mathrm{o}}$ is the dark fluorescence from antennas with all RCs closed, i.e., with the primary quinone acceptor $\mathrm{Q}_{\mathrm{A}}$ fully oxidized. The non-variable fluorescence level at the onset of excitation, measured by a few non-actinic measuring flashes before excitation, usually is $20-25 \%$ above $F_{\mathrm{o}}$ due to the fact that (see also Fig. 2) $10-12.5 \%$ of the RCs in 10 min dark-adapted chloroplasts are in a single reduced state and contain $\mathrm{Q}_{\mathrm{A}}^{-}$. STFs were found to be saturating as concluded from the constancy of the relative fluorescence signal $F_{\mathrm{m}} / F_{\mathrm{o}}$ upon $50 \%$ decrease in flash intensity, or alternatively in chloroplast density. Further details about the use of this equipment can be found elsewhere (Nedbal et al. 1999, and see also http:// www.psi.cz).

We have applied and studied the fluorescence response upon what we call 'twin'-single turnover flashes $\left(\mathrm{T}_{\Delta t} \mathrm{TF}\right)$. A $\mathrm{T}_{\Delta t} \mathrm{TF}$, or in short hand notation TTF (twin), is composed of two identical STFs $\left(\mathrm{STF}_{1}\right.$ and $\left.\mathrm{STF}_{2}\right)$ which are separated by a darktime interval $\Delta t$ (in $\mu \mathrm{s}$ ) in the range between 10 and $500 \mu \mathrm{s}$. The idea behind application of TTFs is the following. STF $_{1}$ (i.e., the first partner of the twin) causes single reduction of the acceptor pair $\left[\mathrm{PheQ}_{\mathrm{A}}\right]$ associated with a change in fluorescence with amplitude $F_{\mathrm{m}}^{\mathrm{STF}}\left(\sim 3^{*} F_{\mathrm{o}}\right)$. The second twin partner $\mathrm{STF}_{2}$ is meant to reach the $\mathrm{RC}$ when its acceptor pair is still partly reduced, i.e., with a fraction in state $\left[\mathrm{PheQ}_{\mathrm{A}}^{-}\right]$. As the reoxidation of $\mathrm{Q}_{\mathrm{A}}^{-}$is known to occur in the time range of $250-500 \mu \mathrm{s}$, significant fraction $(>80 \%)$ of $\mathrm{Q}_{\mathrm{A}}^{-}$is present if $\mathrm{STF}_{2}$ is fired within $10-50 \mu$ s after $\mathrm{STF}_{1} . \mathrm{STF}_{2}$ of $\mathrm{T}_{10} \mathrm{TF}$ (or $\mathrm{T}_{20} \mathrm{TF}$ ) then might cause a double reduction of the acceptor pair, resulting in $\left[\mathrm{Phe}^{-} \mathrm{Q}_{\mathrm{A}}^{-}\right]$or $\left[\mathrm{PheQ}_{\mathrm{A}}\right]^{2-}$. Dependent on its actual lifetime, the double reduction by $\mathrm{T}_{\Delta t} \mathrm{TF}$ is expected to be associated with a further increase in $F_{\mathrm{m}}$ with $\Delta F_{\mathrm{m}}=F_{\mathrm{m}}^{\mathrm{TTF}}-F_{\mathrm{m}}^{\mathrm{STF}}\left(=F_{\mathrm{m}}^{\mathrm{STF} 2}-F_{\mathrm{m}}^{\mathrm{STF} 1}\right)$. An increase of this kind has been shown for a 2nd STF in the presence of DCMU (Vredenberg et al. 2006, Fig. 3). The term 'twin' $\left(\mathrm{T}_{\Delta t} \mathrm{TF}\right)$ is restricted here, unless indicated otherwise, for two subsequent STFs with $\Delta t<500 \mu \mathrm{s}$. In the other cases they form elements of a train of STFs. Exponential decomposition and quadratic least square fitting of the fluorescence decay were done (see also Vredenberg et al. 2006) with standard routines provided by appropriate software (MathCad 13, MathSoft Inc. Cambridge, MA). 


\section{Results and interpretation}

Figure 1 shows the release (upward moving trace) and dark recovery of chl fluorescence quenching upon excitation of a (5-10 min) dark-adapted thylakoid preparation, isolated from fresh pea leaves, with a $12.5 \mathrm{~Hz}$ train of 2 individual STFs (i.e., with dark interval $80 \mathrm{~ms}$ ) and a twin-single turnover flash $\left(\mathrm{T}_{50} \mathrm{TF}\right)$. The first partner of the twin $\left(\mathrm{STF}_{1}\right.$, and identical to the 1 st STF in the train) causes a fluorescence increase with maximum (relative to $F_{\mathrm{o}}=1$ ) $F_{\mathrm{m}}^{\mathrm{STF}} \sim 2.9$. This value agrees with single turnover $F_{\mathrm{m}}$ values reported by others for a variety of plant chloroplasts and algae (Samson and Bruce 1996; Koblizek et al. 2001; Vredenberg et al. 2005). It corresponds with a maximal variable fluorescence in a STF equal to $F_{\mathrm{v}}^{\mathrm{STF}} \sim 1.9 * F_{\mathrm{o}}$.

The $\mathrm{T}_{50} \mathrm{TF}$ (twin) induces a fluorescence increase with, for the present experiment, a maximum $F_{\mathrm{m}}^{\mathrm{T}_{50} \mathrm{TF}} \sim 3.3 * F_{\mathrm{o}}$, which is $\sim 15 \%$ above $F_{\mathrm{m}}^{\mathrm{STF}_{1}}$. The difference between the maximal fluorescence in $\mathrm{T}_{\Delta t} \mathrm{TF}$ - and STF-excitation is defined as the increase in variable fluorescence $\Delta F_{\mathrm{v}}=\Delta F_{\mathrm{m}}=F_{\mathrm{m}}^{\mathrm{T}_{\Delta t} \mathrm{TF}}-F_{\mathrm{v}}^{\mathrm{STF}}$, caused by a TTF in darkadapted chloroplasts. The maximal fluorescence in the 2 nd STF of the train, fired $80 \mathrm{~ms}$ after $\mathrm{STF}_{1}$, is $F_{\mathrm{m}}^{\mathrm{STF}_{2}} \sim 3.4 * F_{\mathrm{o}}$ which, although small, is distinctly higher than $F_{\mathrm{m}}^{\mathrm{T}_{50} \mathrm{TF}}\left(\sim 3.3 * F_{\mathrm{o}}\right)$. It is reproducibly found for dif-

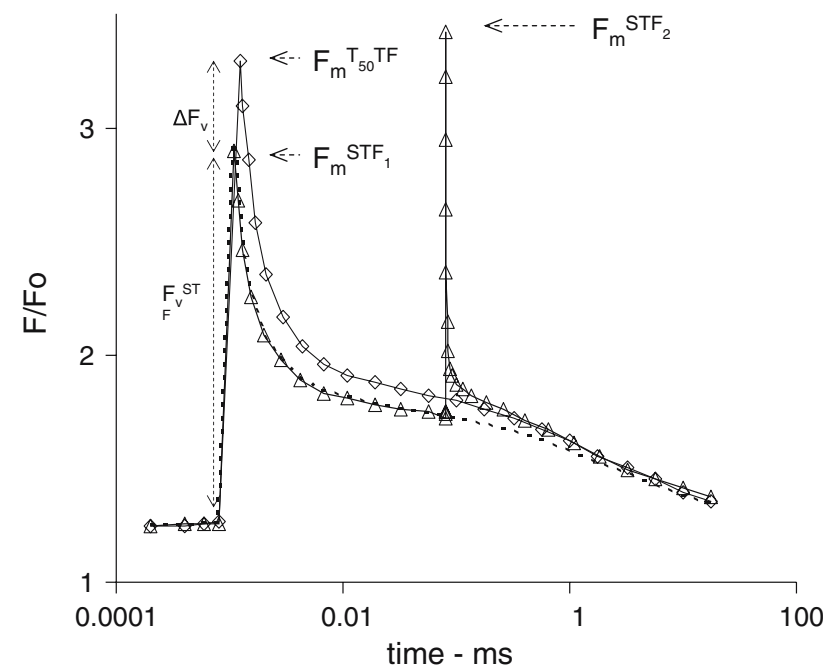

Fig. 1 Fluorescence response $F(t)$ of $10 \mathrm{~min}$ dark-adapted pea thylakoids in the $30 \mu \mathrm{s}$ to $18 \mathrm{~s}$ time range (logarithmic scale) to a $12.5 \mathrm{~Hz}$ train of 1 and 2 STFs ( $35 \mu$ s duration) and to a twin turnover flash $\left(\mathrm{T}_{50} \mathrm{TF}\right)$, plotted relative to the fluorescence $F_{\mathrm{o}}(=1)$ at the onset of the first flash. $F_{\mathrm{m}}$ is the maximum F-level after each STF or TTF. The first partner $\left(\mathrm{STF}_{1}\right)$ of the $\mathrm{T}_{50} \mathrm{TF}$ (twin) is identical to the 1st STF in the train, the 2nd partner $\left(\mathrm{STF}_{2}\right)$ is fired $50 \mu \mathrm{s}$ after $\mathrm{STF}_{1}$. Note (i) $\sim 20 \%$ increase in $F_{\mathrm{m}}$ induced by $\mathrm{T}_{50} \mathrm{TF}$ as compared to that induced by $\mathrm{STF}_{1}$, and (ii) $\sim 30 \%$ increase in $F_{\mathrm{m}}$ induced by 2 nd $\mathrm{STF}$ in $12.5 \mathrm{~Hz}$ train as compared to that induced by $\mathrm{STF}_{1}$. It has been assumed that $\sim 10 \%$ of RCs in the dark-adapted sample is in the single reduced state (see Materials and methods) ferent thylakoid preparations that $F_{\mathrm{m}}^{\mathrm{STF}_{2}}$ (in a train) $>F_{\mathrm{m}}^{\mathrm{T}_{\Delta t} \mathrm{TF}}$. This suggests that the response of the fraction of $\mathrm{Q}_{\mathrm{B}}$-reducing ('normal') $\mathrm{RCs}$ is higher in the 2nd of the first two subsequent STFs in a train. This is in agreement with the documented binary oscillation of fluorescence associated with the two-electron gate mechanism at the acceptor side of PSII (Bowes and Crofts 1980; Shinkarev 1996, 2004). The dark recovery of the quenching after an STF is multiphasic and, as shown by many others, extends over more than four decades (Urban et al. 1999; Roberts et al. 2003; Vredenberg et al. 2006).

Figure 2 shows the experimental and calculated fluorescence decay curves in the dark after one STF $\left(\mathrm{STF}_{1}\right)$ in 10 min dark-adapted thylakoids. The quenching recovery during the so-called $F$ (ast)-phase in the $10 \mu$ s to $100 \mathrm{~ms}$ time range has been shown to originate from two distinguishable RC fractions, variable in size. One fraction contains $\mathrm{Q}_{\mathrm{B}}$-nonreducing $\mathrm{RCs}$, in which the acceptor has

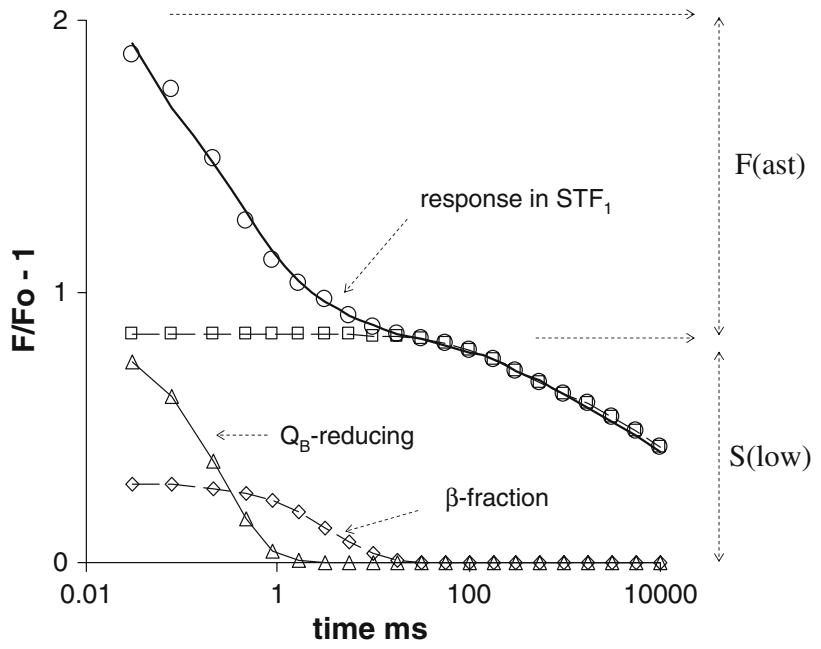

Fig. 2 Experimental (upper solid curve) and calculated (symbols) fluorescence dark decay in 5-10 min dark-adapted chloroplasts after one STF in the $50 \mu$ s to $20 \mathrm{~s}$ time range plotted on a log time scale. Calculated curves were derived for the relatively fast $(<10 \mathrm{~ms})$ and slow phases $\mathrm{F}$ and $\mathrm{S}$, respectively of the multiphasic decay, using a quadratic least square fitting routine and the equations given below. The F-phase can be deconvoluted with two kinetically distinguishable functions $F^{\mathrm{sc}}(t)$ and $F_{\mathrm{nQb}}^{\mathrm{c}}(t) . F_{\mathrm{nQb}}^{\mathrm{c}}(t)$ is of the so-called $\beta$-fraction and attributed to $\mathrm{Q}_{\mathrm{B}}$-nonreducing RCs with double reduced acceptor pair (open diamonds); $F^{\mathrm{sc}}(t)$ is of the complementary fraction with 'normal' single reduced $\mathrm{Q}_{\mathrm{B}}$-reducing RCs (triangles). The S-phase follows a bi-exponential function $F_{\mathrm{nQb}}^{\mathrm{c}}(t)$, attributed to the slow re-oxidation of $\mathrm{Q}_{\mathrm{A}}^{-\mathrm{S}}$ in single reduced $\mathrm{Q}_{\mathrm{B}}$-nonreducing $\mathrm{RCs}$ (open squares). The overall fit is given by the open circles. The following equations apply for the distinguished fractions (see also Vredenberg e t a 1. 2006$)$ : $\quad F^{\mathrm{sc}}(t)=\Delta F^{\mathrm{Qa}}\left(\mathrm{e}^{-k_{\mathrm{AB} 1} t}+\mathrm{e}^{-k_{\mathrm{AB} 2} t}\right), \quad F_{\mathrm{nOb}}^{\mathrm{c}}(t)=$ $\Delta F^{\mathrm{Phe}}\left(\mathrm{e}^{-k_{1} t}+\mathrm{e}^{-k_{2} t}\right) \quad F_{\mathrm{nQb}}^{\mathrm{sc}}(t)=\Delta F^{\mathrm{nQb}}\left(\mathrm{e}^{-k_{\mathrm{nB} 1} t}+\mathrm{e}^{-k_{\mathrm{nB} 2} t}\right), \quad$ w i t h $\Delta F^{\mathrm{Qa}}=\Delta F^{\mathrm{Phe}}=\Delta F^{\mathrm{nQ}}=0.5 * F_{\mathrm{m}}^{\mathrm{STF}_{1}}$ The mean deviation for the individual data points in each of the curves is found to be less than $1 \%$. The approximate parameter values in this STF-experiment were $F_{\mathrm{m}}^{\mathrm{STF}} / F_{\mathrm{o}} \sim 2.9$ and $k_{\mathrm{AB} 1}\left(\sim k_{\mathrm{AB} 2}\right), k_{1}, k_{2}, k_{\mathrm{nB} 1}$, and $k_{\mathrm{nB} 2} \sim 3.6,0.5,0.2$, $3 \times 10^{-3}$, and $10^{-4} \mathrm{~ms}^{-1}$, respectively (see also legend of Fig. 3) 
become double reduced (with $\left[\mathrm{PheQ}_{\mathrm{A}}\right]^{2-}$ ). Its variable size is designated with $\beta$. The fraction is in short hand notation called the $\beta$-fraction with double reduced $\mathrm{Q}_{\mathrm{B}}$-nonreducing RCs. The other responsive fraction contains single reduced 'normal' RCs (Vredenberg et al. 2006). The quenching recovery during the F-phase is superimposed on a quasistationary background quenching. The latter decays, in rough approximation, bi-exponentially in the so-called $\mathrm{S}$ (low)-phase in the $50 \mathrm{~ms}$ to $20 \mathrm{~s}$ time range. It is attributed to quenching recovery of RCs filled with $\mathrm{Q}_{B}$-nonreducing $\mathrm{RCs}$, in which a slow re-oxidation of $\mathrm{Q}_{\mathrm{A}}^{-}$takes place.

Fitting was done assuming exponential recoveries associated with reoxidation of the single and double reduced acceptor pair each with their specific rate constants. Details of the procedure are given in a previous paper (Vredenberg et al. 2006, but see also the legend of Fig. 3). If we take $F_{20 \mathrm{~ms}}-F_{\mathrm{o}}$ as the approximate fractional size $\gamma$ of the S-phase we find for this dark-adapted thylakoid preparation, in fair agreement with results reported before, $\gamma \sim 0.4$. Quenching recovery in the single reduced 'normal' RCs occurs with rate constants in the range between 4 and $1 \mathrm{~ms}^{-1}$ which is in the range reported for the re-oxidation of $\mathrm{Q}_{\mathrm{A}}^{-}$formed by $\mathrm{STF}_{1}$. The analysis shows further that the size of the $\beta$-fraction with double reduced $\mathrm{Q}_{\mathrm{B}}$-nonreducing $\mathrm{RCs}$ is $\beta \sim 20 \%$. These in turn constitute under these darkadapted conditions $\sim 11 \%$ of the total population of excited RCs as concluded from the amplitude of $F_{\mathrm{v}}$. Note that $\sim 26 \%$ of the $\mathrm{S}$-recovery phase is likely to originate from the $\mathrm{Q}_{\mathrm{B}}$-nonreducing $\beta$-fraction of excitable RCs.

Figures $3 \mathrm{~A}$ and $\mathrm{B}$ (with for A the same data as in Fig. 2) show, on a linear $0-10 \mathrm{~ms}$ time scale, the kinetics of the recovery of STF- and TTF-induced quenching release, respectively in the two subfractions of the F-phase (see also Fig. 2). The analysis shows that the size of the $\beta$-fraction with double reduced $\mathrm{Q}_{\mathrm{B}}$-nonreducing $\mathrm{RCs}$ increases from $\beta=26$ to $40 \%$, without a significant change in the quenching recovery rate constant in the range $0.5-0.2 \mathrm{~ms}^{-1}$. The recovery rate constant of the decreasing fraction of single reduced $\mathrm{Q}_{\mathrm{B}}$-reducing $\mathrm{RCs}$ is in the range of 3-5 $\mathrm{ms}^{-1}$. The magnitude of the S-phase after $\mathrm{T}_{20} \mathrm{TF}$-excitation, as compared to that in STF has slightly increased (from 44 to $46 \%$ ). It is noteworthy that the size of the fraction of $\mathrm{Q}_{\mathrm{B}}$-reducing RCs is approximately the same in both excitations. This is what would be expected because STF- and TTF flashes are saturating with respect to trapping activity. Figure $3 \mathrm{C}$ shows experimental and calculated kinetics of the difference in response between $\mathrm{T}_{20} \mathrm{TF}$ - and $\mathrm{STF}_{1}$-excitations. It illustrates in detail that the different response originates exclusively from the $\beta$-fraction with almost identical recovery rate constants as in a single turnover flash.

Figure 4 shows the $F(t)$ curves in the first four STFs in a $12.5 \mathrm{~Hz}$ train and those (dashed curves) in which the last flash in a train of variable length $N(N=1,2,3,4)$ was replaced by a $\mathrm{T}_{50} \mathrm{TF}$. It shows that the $\mathrm{T}_{50} \mathrm{TF}$-induced increase in $F_{\mathrm{m}}$ is modulated with flash number of the 1 st

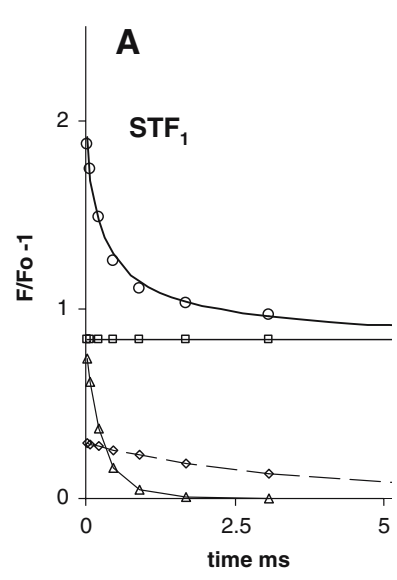

Fig. 3 Experimental (upper solid curves) and calculated fluorescence decay curves (symbols) in the dark after one STF (A), one $\mathrm{T}_{20} \mathrm{TF}(\mathbf{B})$, and (C) for the difference response of $\mathbf{B}$ and $\mathbf{A}$ (= TTF$\mathrm{STF}$ ) in the linear 0-10 ms time range. Calculated curves (symbols) were derived as outlined in the legend of Fig. 2. Parameter values corresponding with the calculated curve of $\mathbf{A}-\mathbf{C}$ are given below;

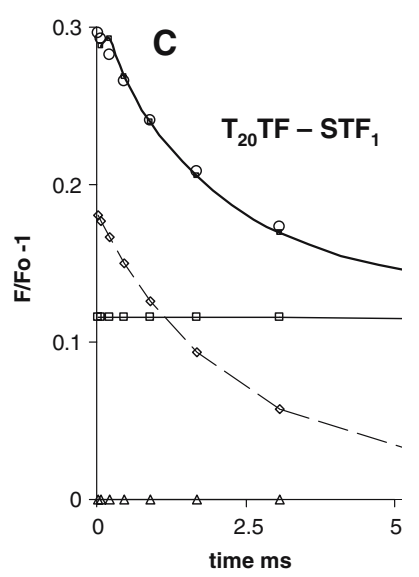

rate constants $(\mathrm{K})$ are given in $\mathrm{ms}^{-1}$. The 2 nd exponential in $F^{\mathrm{sc}}(t)$ is assumed not to be involved (n.i.), assuming that the fraction in the dark-adapted preparation complementary to the $\beta$-fraction is $100 \%$ $\mathrm{S}_{1}$ with $\mathrm{Q}_{\mathrm{B}}$ fully oxidized. The mean deviation for the individual data points in each of the curves is found to be less than $1 \%$

\begin{tabular}{|l|c|c|c|c|c|c|c|c|}
\hline excitation & $\mathrm{F}_{\mathrm{m}} / \mathrm{F}_{\mathrm{o}}$ & $\beta$ & $\mathrm{k}_{\mathrm{AB} 1}$ & $\mathrm{k}_{\mathrm{AB} 2}$ & $\mathrm{k}_{1}$ & $\mathrm{k}_{2}$ & $\mathrm{k}_{\mathrm{nB} 1}\left(10^{3}\right)$ & $\mathrm{k}_{\mathrm{nB} 2}\left(10^{4}\right)$ \\
\hline $\mathrm{STF}(\mathrm{A})$ & 2.9 & 0.3 & 3.6 & n.i. & 0.5 & 0.2 & 3.4 & 1 \\
\hline $\mathrm{T}_{20} \mathrm{TF}(\mathrm{B})$ & 3.2 & 0.4 & 3.6 & n.i. & 0.5 & 0.2 & 3.7 & 1 \\
\hline $\mathrm{T}_{20} \mathrm{TF}-\mathrm{STF}(\mathrm{C})$ & 1.3 & 1 & n.i. & n.i. & 0.6 & 0.2 & 2.8 & 1 \\
\hline
\end{tabular}


Fig. $4 F(t)$ curves in the first four STFs in a $12.5 \mathrm{~Hz}$ train and those in which the last flash in this train of variable length $N$ $(N=1,2,3,4)$ was replaced by a $\mathrm{T}_{100} \mathrm{TF}$. The curves are on a linear time scale in the $0-5 \mathrm{~ms}$ domain. $\mathrm{S}(\mathrm{T}) \mathrm{TFs}$ for $N=2,3$, and 4 are fired 80,160 , and $240 \mathrm{~ms}$, respectively after the first excitation. Note the increased $F$ level at the start of these $\mathrm{S}(\mathrm{T}) \mathrm{TFs}$ as compared to that at the first excitation


partner of the TTF (twin) with maximum and minimum for $N=1$ and 3, respectively and strongly reduced increases for $N=2$ and 4 . The absence of a TTF-induced increase in the 3rd flash confirms that the STFs are saturating with respect to trapping activity and associated release of fluorescence quenching.

Figure 5A illustrates, in the linear $0-5 \mathrm{~ms}$ time range, the $F(t)$ response of a $5 \mathrm{~min}$ dark-adapted chloroplast preparation to 1,2 , and $4 \mathrm{kHz}$ trains of 40 or 60 STFs. The curves show for each train a reproducible $F_{\mathrm{m}}$ in $\mathrm{STF}_{1}$ $\left(F_{\mathrm{m}}^{\mathrm{STF}_{1}}\right)$ with $F_{\mathrm{m}}^{\mathrm{STF}_{1}} \sim 3 * F_{\mathrm{o}} . F_{\mathrm{m}}$ increases after the first flash with a maximum reached at the 3 rd flash $\left(\mathrm{STF}_{3}\right)$ of the train with, for this preparation, $F_{\mathrm{m}}^{\mathrm{STF}_{3}} \sim 3.6 * F_{\mathrm{o}}$ for a $4 \mathrm{kHz}$ train. The $\sim 20 \%$ increase in $F_{\mathrm{m}}$ above $F_{\mathrm{m}}^{\mathrm{STF}_{1}}$ is



Fig. 5 (A) $F(t)$ response of a 5 min dark-adapted chloroplast preparation to a 1 (triangles), 2 (diamonds) and $4 \mathrm{kHz}$ train (squares) of 40 or 60 STFs in the linear $0-5 \mathrm{~ms}$ time range. $F_{\mathrm{m}}$ in 1 st STF is $F_{\mathrm{m}}^{\mathrm{STF}_{1}} \sim 3 * F_{\mathrm{o}}$. Increase of $F_{\mathrm{m}}$ in the next flashes of the train reaches a maximum at the 3rd flash $\left(\mathrm{STF}_{3}\right)$ with, for this preparation, $F_{\mathrm{m}}^{\mathrm{STF}_{3}} \sim 3.6 * F_{\mathrm{o}}$. Subsequently $F_{\mathrm{m}}$ declines transiently in the next 4 and 8 STF excitations in the 2 and $4 \mathrm{kHz}$ flash train, transient and declines in the next $2 \mathrm{~ms}$ of 4 and 8 STF excitations in a 2 and $4 \mathrm{kHz}$ flash train, respectively reaching a dip D approx. $3 \mathrm{~ms}$ after the onset of the flash train. The increase in $F_{\mathrm{m}}^{\mathrm{STF}}$, as shown for TTF excitation (Figs. 1-4), is caused by electron trapping in, in this case, a $30 \% \beta$-fraction of $\mathrm{Q}_{\mathrm{B}}$-nonreducing RCs. The transient decline in $F_{\mathrm{m}}^{\mathrm{TTF}}$ is likely to be caused, as will be discussed below, by reoxidation of the double reduced acceptor pair in the $\mathrm{Q}_{\mathrm{B}}$-nonreducing $\mathrm{RCs}$. We have seen that this reduction occurs with a rate constant in the range between 0.5 and $0.2 \mathrm{~ms}^{-1}$, i.e., in the $2-5 \mathrm{~ms}$ time range (Fig. 4). Figure 5B gives the $F(t)$ response to the flash trains in the 0-60 ms time range on a logarithmic scale. It shows, after the transient response during the initial $3 \mathrm{~ms}$ STF-excita-

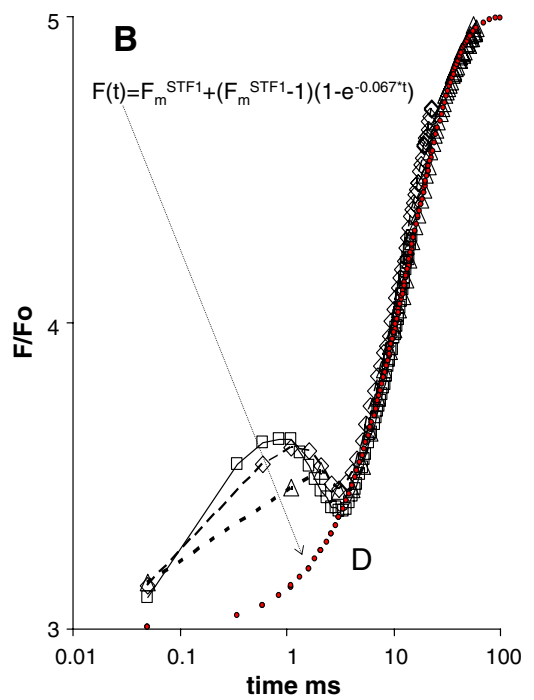

respectively towards a dip D. A subsequent increase in $F_{\mathrm{m}}^{\mathrm{STF}}$ occurs after approx. $3 \mathrm{~ms}$ of ongoing excitations independent of the frequency of the train. (B) Same responses in the 0-60 ms range plotted on a logarithmic time scale. It shows the gradual rise in $F_{\mathrm{m}}$ with, for the $4 \mathrm{kHz}$ train of $60 \mathrm{STFs}$, a saturation level $F_{\mathrm{m}}^{\mathrm{STF}} \sim 5 * F_{\mathrm{o}}$. The dotted curve is the one for the exponential curve $F(t)=F_{\mathrm{m}}^{\mathrm{STF}_{1}}-\left(1-F_{\mathrm{m}}^{\mathrm{STF}_{1}}\right) *\left(1-\mathrm{e}^{-0.07 * t}\right)$ 
tion period, a gradual rise in $F_{\mathrm{m}}$ with, for a $1 \mathrm{kHz}$ train of 60 STFs, a saturation level $F_{\mathrm{m}}^{\mathrm{STF}_{60}} \sim 5 * F_{\mathrm{o}}$. This rise, as has been shown (Vredenberg et al. 2006), is due to quenching release associated with double reduction of accumulating single reduced $\mathrm{Q}_{\mathrm{B}}$-nonreducing RCs. It appears that the rise in the 3 - to $60-\mathrm{ms}$ time range is independent of the frequency of the STF train in the $1-4 \mathrm{kHz}$ range. Moreover, the $F_{\mathrm{m}}$ rise from $F_{\mathrm{m}}^{\mathrm{STF}_{1}}\left(\sim 3 * F_{\mathrm{o}}\right)$ is close to that of an exponential function $F(t)=F_{\mathrm{m}}^{\mathrm{STF}_{1}}-\left(1-F_{\mathrm{m}}^{\mathrm{STF}_{1}}\right) *\left(1-\mathrm{e}^{-k * t}\right) \quad$ with $k \sim 0.067 \mathrm{~ms}^{-1}$. This suggests that, in rough approximation, the triggering reaction for closing of all RCs occurs with a turnover relaxation time of $\sim 15 \mathrm{~ms}$.

Logarithmic time plots of the $F(t)$ response to a $12.5 \mathrm{~Hz}$ train in the absence and to a $12.5 \mathrm{~Hz}$ and $2 \mathrm{kHz}$ train of 60 STFs in the presence of $2 \mu \mathrm{M}$ FCCP are reproduced in Fig. 6. They show (i) a rise in $F_{\mathrm{m}}$ from $F_{\mathrm{m}}^{\mathrm{STF}_{1}} \sim 3 * F_{\mathrm{o}}$ to $F_{\mathrm{m}}^{\mathrm{STF}_{60}} \sim 5 * F_{\mathrm{o}}$ which, for a $12.5 \mathrm{~Hz}$ train and in agreement with earlier results, is inhibited by FCCP; (ii) inhibition of the $F_{\mathrm{m}}$ rise by FCCP is not observed in a $2 \mathrm{kHz}$ train, and (iii) the transient increase in $F_{\mathrm{m}}$ during the first $3 \mathrm{~ms}$ of 7-8 STF excitations in a $2 \mathrm{kHz}$ train is not affected by the ionophores (not shown, but see the control curve in Fig. 5).

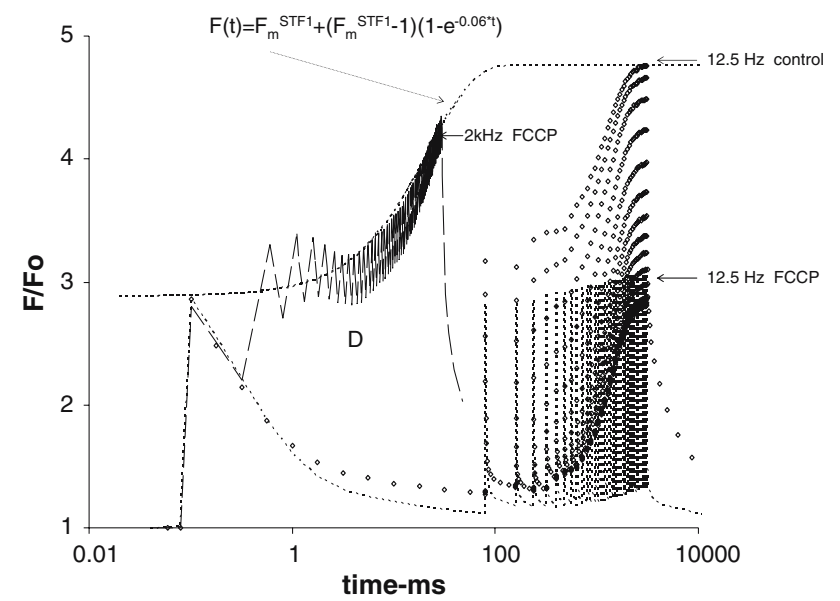

Fig. 6 Logarithmic time plots of the $F(t)$ response to a $12.5 \mathrm{~Hz}$ train of 60 STFs in absence (open diamonds) and presence of $2 \mu \mathrm{M}$ FCCP (lower dotted curve) and, in the presence of the ionophore, to the same train at $2 \mathrm{kHz}$ (dashed curve). The response in the $12.5 \mathrm{~Hz}$ train shows the commonly observed pattern with increase in $F_{\mathrm{m}}$ from $F_{\mathrm{m}}^{\mathrm{STF}_{1}} \sim 2.9 * F_{\mathrm{o}}$ to $F_{\mathrm{m}}^{\mathrm{STF}_{60}} \sim 5 * F_{\mathrm{o}}$ which, for a $12.5 \mathrm{~Hz}$ train and in agreement with earlier results, is nearly completely inhibited by FCCP. A qualitatively unperturbed response of the $F_{\mathrm{m}}$ rise is seen in the presence of FCCP in a $2 \mathrm{kHz}$ train with the transient rise and decline in the first 7-8 STF excitations (see for the response in the control Fig. 5). The upper dotted curve is the one for the exponential curve $F(t)=F_{\mathrm{m}}^{\mathrm{STF}_{1}}-\left(1-F_{\mathrm{m}}^{\mathrm{STF}_{1}}\right) *\left(1-\mathrm{e}^{-0.06 * t}\right)$

\section{Discussion}

The increased variable fluorescence $\Delta F_{\mathrm{v}}$ (Fig. 1) associated with double excitation by a TTF (twin) is ascribed to electron trapping in RCs in which, at the time of excitation by the second partner $\left(\mathrm{STF}_{2}\right)$ of the twin, the primary acceptor pair $\left[\mathrm{PheQ}_{\mathrm{A}}\right]$ is in its single reduced state [Phe$\left.\mathrm{Q}_{\mathrm{A}}\right]^{-}$. These data provide experimental evidence that, under proper conditions and concomitantly with exciton trapping in a twin excitation, the acceptor pair of PSII can become double reduced $\left[\mathrm{PheQ}_{\mathrm{A}}\right]^{2-}$. Occurrence of double reduction of the PSII acceptor pair has been suggested before (Doschek and Kok 1972; Joliot and Joliot 1977; France et al. 1992). It has received experimental support from fluorescence measurements in $12.5 \mathrm{~Hz}$ flash trains (Vredenberg et al. 2006). These showed an approximate twofold increase in $F_{\mathrm{m}}$ relative to $F_{\mathrm{o}}$, reflecting the accumulation of a sizeable fraction of excitable $\mathrm{Q}_{\mathrm{B}}$-nonreducing centers in the train (see also Fig. 6). The size of the $\mathrm{T}_{\Delta t} \mathrm{TF}$-induced increase in variable fluorescence $\Delta F_{\mathrm{v}}$ (Fig. 1) was found to be invariable with the darktime interval $\Delta t$ between the partners of the twin in the range between 10 and $200 \mu \mathrm{s}$ (data not shown).

The dark recovery of the fluorescence signal after $\mathrm{T}_{\Delta t} \mathrm{TF}$-excitation is distinctly different from that after STFexcitation (Figs. 2, 3). The major recovery phase of the complementary component of the fluorescence quenching release activated by the twin occurs, as deduced after curve fitting (Fig. 3), exponentially with two approximately equally weighed amplitudes and rate constants $k_{1} \sim 400$ and $k_{2} \sim 200 \mathrm{~s}^{-1}$, respectively and reflects the re-oxidation of the double reduced acceptor pair $\left[\mathrm{PheQ}_{\mathrm{A}}\right]^{2-}$ formed by TTF. These rate constants agree reasonably well with those deduced from the dark recovery of accumulating $\mathrm{Q}_{\mathrm{B}}$-nonreducing centers after their excitation (Vredenberg et al. 2006). Deconvolution of the $F(t)$ curve in TTF excitation (Fig. 3B) shows, as compared to STF excitation (Fig. 3A), an increased $\beta$ fraction which amounts $60-65 \%$ of the $S$ phase of the recovery phase. It is unknown how the remaining $35-40 \%$ of the $\mathrm{Q}_{\mathrm{B}}$-nonreducing $\mathrm{RCs}$ in this segment behave upon double excitation. Their behavior and function remain to be established. It might be that the trapping efficiency and life time of the double reduced acceptor pair in these RCs are beyond their limit for giving rise to a release of fluorescence quenching upon excitation.

Figure 4 shows a particular aspect of the supplemental increase in variable fluorescence $\Delta F_{\mathrm{v}}$ upon $\mathrm{T}_{\Delta t} \mathrm{TF}$ excitation. The $F(t)$ response, in this case for $\mathrm{T}_{50} \mathrm{TF}$, is monitored for a twin-excitation which is preceded by a $12.5 \mathrm{~Hz}$ train of STFs, variable in number $N$ and shown here for $0 \leq N \leq 3$. It shows, in dependence of the number $(N)$ of preceding STFs, a modulated pattern of $\Delta F_{\mathrm{v}}$ with maximum at $N=0$ and 3. The modulation depth of $\Delta F_{\mathrm{v}}$ is 
substantially larger than that of $F_{\mathrm{v}}^{\mathrm{STF}}$ seen in the train of STFs. This behavior points to a dependence of $\Delta F_{\mathrm{v}}$ on the PSII electron trapping efficiency $\Phi_{\mathrm{tr}}$ in the 2nd excitation of the TTF. This efficiency $\phi_{\text {tr }}$ is determined, under conditions at which $\mathrm{Q}_{\mathrm{A}}$ is reduced (Vredenberg 2004), by the rate constant $k_{\mathrm{y}}$ of electron transfer from $\mathrm{Y}_{\mathrm{z}}$ to $\mathrm{P}^{+}{ }_{680}$ at the PSII donor side, in competition with the rate constant $k_{-1}$ of radical pair recombination in the RC with $\phi_{\mathrm{tr}}=k_{\mathrm{y}} / k_{\mathrm{y}}+k_{-1}$. This gives, with established values (Roelofs et al. 1992; Meyer et al. 1989; Schilstra et al. $1998)$ for $k_{-1}\left(\sim 3 \times 10^{5} \mathrm{~ms}^{-1}\right.$, independent of $\mathrm{S}$ states $)$ and $k_{\mathrm{y}}$ (S-state dependent) $\phi_{\mathrm{tr}}$ values of about 0.8 for $\mathrm{S}_{1}$ and $\mathrm{S}_{2}$, 0.05 and $<0.01$ for $S_{3}$ and $S_{4(0)}$, respectively. A similar but attenuated pattern is found for $4 \leq N \leq 7$ (data not shown). This indicates that the modulation of $F_{\mathrm{v}}$ in TTFs preceded by N STFs occurs with a period-of-four oscillation. The modulation pattern of $\Delta F_{\mathrm{v}}$ is in fair agreement with electron transport regulation at the $\mathrm{Y}_{\mathrm{z}}$ to $\mathrm{P}_{680}^{+}$donor side of PSII, if it is assumed that chloroplasts, dark-adapted for tens of minutes, have as an average a $S_{1} / S_{0} \sim 0.75 / 0.25$ heterogeneity (Vermaas et al. 1984). Whether or not, and if so to what extent the modulation pattern will change in preparations that have been dark-adapted for several hours remains to be established. These preparations were shown to have an altered $S_{0} / S_{1}$ heterogeneity (Toth et al. 2005).

It has been illustrated and discussed elsewhere (Hiraki et al. 2003; Vredenberg 2004) that the major part of the approx. 25\% $\mathrm{S}_{0}$-fraction in 5-10 min dark-adapted chloroplast preparations is in a state in which $\mathrm{Q}_{\mathrm{B}}$ is reduced. If we accept that the increase of $F_{\mathrm{m}}$ in a TTF $\left(\Delta F_{\mathrm{v}}^{\mathrm{TTF}}\right)$ in darkadapted chloroplasts is due to release of photochemical quenching associated with double reduction of acceptor pair $\left[\mathrm{PheQ}_{\mathrm{A}}\right]^{2-}$ in single reduced $\mathrm{RCs}$ we come to a following interpretation of these events: (i) The relative size and average lifetime of the TTF-induced increase in variable fluorescence with $\Delta F_{\mathrm{v}}^{\mathrm{TTF}} / F_{\mathrm{v}}^{\mathrm{STF}}$ in the range $20-30 \%$ (Fig. 1) and lifetime 4-5 ms (Fig. 3C) suggests that the lifetime of $\left[\mathrm{PheQ}_{\mathrm{A}}\right]^{2-}$ in $70-80 \%$ of RCs is too short to lead to release of fluorescence quenching; (ii) the strong reduction of $\Delta F_{\mathrm{v}}^{\mathrm{TTF}}$ in TTFs preceded by 1,2 or $3 \mathrm{STFs}$ (Fig. 4) offers evidence that the fraction of RCs in darkadapted chloroplasts which shows a $20-30 \%$ TTF-induced increase in variable fluorescence $\Delta F_{\mathrm{v}}^{\mathrm{TTF}}$ is identical with the $\mathrm{S}_{0}$ fraction, and (iii) the lifetime of $\left[\mathrm{PheQ}_{\mathrm{A}}\right]^{2-}$ in $\mathrm{S}_{1,2,3^{-}}$ fractions is extremely short.

The requirement of 3-4 STFs in high frequency STF trains and the pattern by which the maximum of $\Delta F_{\mathrm{v}}^{\mathrm{TTF}}$ (Fig. 5) is reached indicates that the trapping efficiency $\phi_{\text {tr }}$ in the fraction with single reduced $\mathrm{Q}_{\mathrm{B}}$-nonreducing $\mathrm{RCs}$ is between 0.8 and 0.85 . This fraction has been transferred in dark-adapted chloroplasts from the $S_{0}$ to $S_{1}$ state in an $\mathrm{STF}_{1}$ excitation with $F_{\mathrm{m}}^{\mathrm{STF}_{1}} \sim 3 * F_{\mathrm{o}}$ (see Fig. 2). If we assume that the rate constant of radical pair recombination in the RCs is $k_{-1}=3 \times 10^{5} \mathrm{~ms}^{-1}$ (Roelofs et al. 1992) then $\phi_{\mathrm{tr}}=k_{\mathrm{y}} /\left(k_{\mathrm{y}}+k_{-1}\right)=0.8$ would yield the rate constant of electron transfer from $\mathrm{Y}_{\mathrm{z}}$ to $\mathrm{P}_{680}^{+}$of $k_{\mathrm{y}} \sim 1-2 \mathrm{~ns}^{-1}$ for the single reduced RCs in $\mathrm{S}_{1}$. This is in the range reported for this state (Schilstra et al. 1998). The decline of $F(t)$ in the $0-3 \mathrm{~ms}$ time range after the transient increase by the first STFs of high frequency trains indicates that the double reduction of the reduced acceptor pair in the complementary fraction, originally in the $S_{1}$ state and after single excitation in the $S_{2}$ state, does not cause a release in quenching in the subsequent 5-10 STFs. This is in agreement with the conclusion, drawn from the results of Fig. 4, that electron trapping in reduced $\mathrm{S}_{1,2,3}$-fractions is close to zero due to the short lifetime of $\left[\mathrm{PheQ}_{\mathrm{A}}\right]^{2-}$ in these fractions. The nearly zero electron trapping efficiency in darkadapted $S_{1,2,3}$ fractions in the reduced state causes the absence of charge accumulation in the subsequent $\mathrm{S}$-states. In other words an original $\mathrm{S}_{0} / \mathrm{S}_{1}$ heterogeneity in a darkadapted sample is transferred to $S_{1} / S_{2}$ heterogeneity which is quasi stable during the first $5-10$ STFs in a high frequency train. The decline in $F(t)$ is likely to reflect the reoxidation of $\left[\mathrm{PheQ}_{\mathrm{A}}\right]^{2-}$ that is generated upon double excitation of the original $\beta$-fraction in the $\mathrm{S}_{0}$ state. The transient $F(t)$ rise and decline in the 0.01-2 ms time range strongly resembles the O-J-D(ip)-I curve in high intensity MTFs in chloroplasts (Schreiber and Neubauer 1987: Strasser et al. 1995), algae (Koblizek et al. 2001) and intact leaves (Schansker et al. 2006). There it shows up as a distinct dip D between the (shifted) $\mathrm{J}$ level and the I level in the OJIP induction curve. The decline in the J-D phase has been interpreted (Schansker et al. 2006) as a transient limitation on the donor side associated with $\mathrm{P}_{680}^{+}$formation. This is at variance with the interpretation given here. Moreover, temporary accumulation of $\mathrm{P}_{680}^{+}$in this time range is difficult to imagine under conditions in which, at the excitation rates that were applied, radical pair recombination is assumed to occur efficiently.

The $F(t)$ rise in the 3-60 ms time range in high frequency flash trains (Fig. 5B) is similar as shown before in a $12.5 \mathrm{~Hz}$ train (Vredenberg et al. 2006). It has been interpreted to be due the photo-electrochemical control of the rate constant of $\left[\mathrm{PheQ}_{\mathrm{A}}\right]^{-}$re-oxidation in $\mathrm{S}_{1,2,3}$-fractions, causing the $\mathrm{pH}\left(\Delta \mu_{\mathrm{H}}\right)$-dependent accumulation of trapping competent $\mathrm{Q}_{\mathrm{B}}$-nonreducing RCs. The double reduction of these RCs causes a further quenching release. The approx. $3 \mathrm{~ms}$ delay of this accumulation, independent of the flash frequency (Fig. 5B), suggests that the onset of photoelectrochemical control of the accumulation is triggered by the proton uptake associated with the protonation of $\mathrm{Q}_{\mathrm{B}}^{2-}$. This apparently requires completion of two turnovers of $\mathrm{Q}_{\mathrm{A}}$ reduction at the acceptor side of PSII in the fraction with $\mathrm{Q}_{\mathrm{B}}$-reducing RCs. This will take $2-3 \mathrm{~ms}$, as illustrated in Fig. 2 and in agreement with documented data (Robinson 
and Crofts 1983). The fact that the onset of the triggering occurs with a delay similar to that of $\mathrm{Q}_{\mathrm{B}}^{2-}$ formation is in harmony with an established fast protonation of $\mathrm{Q}_{\mathrm{B}}^{2-}$ (Haumann and Junge 1994).

The photo-electrochemical control of the $F(t)$ rise in a low frequency flash train is confirmed. The $F(t) / F_{\mathrm{o}}$ rise from 3 to 5 in the $0.5-5 \mathrm{~s}$ time range in a $12.5 \mathrm{~Hz}$ flash train is inhibited, as has been shown before (Vredenberg et al. 2006), by FCCP. If the frequency of the train is increased to $2 \mathrm{kHz}$, i.e., when the dark interval between STFs in a train is reduced from $80 \mathrm{~ms}$ to $500 \mu \mathrm{s}$, the inhibition by the protonophore is released. The recovery of the STFinduced $\mathrm{pH}$ gradient in the presence of FCCP apparently takes more than $500 \mu \mathrm{s}$. Titration of the $F(t) / F_{\mathrm{o}}$ response against the frequency of the flash train in the absence and presence of protonophores will allow the determination of the time constants for the generation and the decay of the event, probably the transmembrane $\Delta \mu_{\mathrm{H}}$, that triggers the equilibrium constant of the $\mathrm{Q}_{\mathrm{A}}^{-} \mathrm{Q}_{\mathrm{B}} \leftrightarrow \mathrm{Q}_{\mathrm{A}} \mathrm{Q}_{\mathrm{B}}^{-}$equilibrium and consequently the accumulation of $\mathrm{Q}_{\mathrm{B}}$-nonreducing RCs (Vredenberg et al. 2006). This has been discussed to cause the doubling of the variable fluorescence. The data of Figs. 5 and 6 indicate that generation occurs, in rough approximation with a protonophore-independent time constant $\tau=55-65 \mathrm{~s}^{-1}$. It is clear that the occurrence of an $F(t)$ rise in a flash train is dependent on (i) the frequency of the train, and (ii) on the proton conductance of the membrane. The absence of such rise in some algae might be caused by an imbalance between these two constraints.

Our results demonstrate that PSII uniquely operates as a two photon and electron trap. The rise of the variable chl $a$ fluorescence in a twin (saturating) single turnover flash (TTF) and in flash trains of 'tuned' frequency, has farreaching consequences for current views on PSII heterogeneity concerning characteristics and properties of PSUs different in antenna size or in electron transport activity. These will require detailed attention in the future.

Acknowledgements This research was supported by the Ministry of Education of the Czech Republic (Research concept MSM6007665808), by the Academy of Sciences of the Czech Republic (Research concepts AV0Z50510513 and AV0Z50200510), and by the Grant Agency of the Czech Republic (Project GACR 206/ 05/0335). We thank V. Kasalicky for assistance with instrument and J. Hunalova for preparation of thylakoids.

\section{References}

Bouges-Bocquet B (1980) Kinetic models for the electron donors of photosystem II of photosynthesis. Biochim Biophys Acta 594:85-103

Bowes JM, Crofts AR (1980) Binary oscillations in the rate of reoxidation of the primary acceptor of Photosystem II. Biochim Biophys Acta 590:373-384
Butler WL (1972) On the primary nature of fluorescence yield changes associated with photosynthesis. Proc Natl Acad Sci USA 69:3420-3422

Doschek WW, Kok B (1972) Photon trapping in photosystem II of photosynthesis. Biophys J 12:832-838

Durchan M, Va'cha F, Krieger-Liszkay A (2001) Effects of severe $\mathrm{CO}_{2}$ starvation on the photosynthetic electron transport chain in tobacco plants. Photosynth Res 68:203-213

Duysens LNM, Sweers HE (1963) Mechanisms of the two photochemical reactions in algae as studied by means of fluorescence. In: Japanese Society of Plant Physiologists (ed) Studies on microalgae and photosynthetic bacteria. University of Tokyo Press, Tokyo, pp 353-372

France LL, Geacintov NE, Breton J, Valkunas L (1992) The dependence of the degrees of sigmoidicities of fluorescence induction curves in spinach chloroplasts on the duration of actinic pulses in pump-probe experiments. Biochim Biophys Acta 1101:105-119

Hankamer B, Barber J, Boekema EJ (1997) Structure and membrane organization of photosystem II in green plants. Annu Rev Plant Physiol Plant Mol Biol 48:641-671

Haumann M, Junge W (1994) The rates of proton uptake and electron transfer at the reducing side of photosystem II in thylakoids. FEBS Lett 347:45-50

Hiraki M, van Rensen JJS, Vredenberg WJ, Wakabayashi K (2003) Characterization of the alterations of the chlorophyll $a$ fluorescence induction curve after addition of photosystem II inhibiting herbicides. Photosynth Res 78:35-46

Joliot P, Joliot A (1977) Evidence for a double hit process in photosystem II based on fluorescence studies. Biochim Biophys Acta 462:559-574

Ke B (2001) Photosynthesis photobiochemistry and photobiophysics. In: Govindjee (ed) Advances in photosynthesis, vol 10. Kluwer Acad Publ, Dordrecht

Klimov VV, Klevanik AV, Shuvalov VA, Krasnovsky AA (1977) Reduction of pheophytin in the primary light reaction of photosystem II. FEBS Lett 82:183-186

Koblizek M, Kaftan D, Nedbal L (2001) On the relationship between the non-photochemical quenching of the chlorophyll fluorescence and the photosystem II light harvesting efficiency. A repetitive flash fluorescence study. Photosynth Res 68:141-152

Kolber ZS, Prasil O, Falkowski PG (1998) Measurements of variable chlorophyll fluorescence using fast repetition rate techniques. I. Defining methodology and experimental protocols. Biochim Biophys Acta 1367:88-106

Kramer DM, Di Marco G, Loretto F (1995) Contribution of plastoquinone quenching to saturation pulse-induced chlorophyll fluorescence in leaves. In: Mathis P (ed) Photosynthesis: from light to biosphere, vol I. Kluwer Acad Publ, Dordrecht, the Netherlands, pp 147-150

Lazár D (2003) Chlorophyll $a$ fluorescence rise induced by high light illumination of dark-adapted plant tissue studied by means of a model of photosystem II and considering photosystem II heterogeneity. J Theor Biol 220:469-503

Lazár D (2006) The polyphasic chlorophyll a fluorescence rise measured under high intensity of exciting light. Funct Plant Biol 33:9-30

Meyer BE, Schlodder E, Dekker JP, Witt HT (1989) $\mathrm{O}_{2}$ evolution and chla $_{\text {II }}^{+}\left(\mathrm{P}_{680}^{+}\right)$nanosecond reduction kinetics in single flashes as a function of pH. Biochim Biophys Acta 974:36-43

Nedbal L, Trtilek M, Kaftan D (1999) Flash fluorescence induction; a novel method to study regulation of photosystem II. J Photochem Photobiol 48:154-157

Papageorgiou G, Govindjee (eds) (2004) Chlorophyll a fluorescence a signature of photosynthesis. Advances in photosynthesis and respiration, vol 19. Springer, Dordrecht 
Roberts AG, Gregor W, Britt RD, Kramer DM (2003) Acceptor and donor-side interactions of phenolic inhibitors of photosystem II. Biochim Biophys Acta 1604:23-32

Robinson HH, Crofts AR (1983) Kinetics of the oxidation reduction reactions of the photosystem II quinone acceptor complex and the path for deactivation. FEBS Lett 151:221-226

Roelofs TA, Lee CH, Holzwarth AR (1992) Global target analysis of picosecond chlorophyll fluorescence kinetics from pea chloroplasts. Biophys J 61:1147-1163

Samson G, Bruce D (1996) Origins of the low yield of chlorophyll fluorescence induced by single turnover flash in spinach thylakoids. Biochim Biophys Acta 1276:147-153

Samson G, Prasil O, Yaakoubd B (1999) Photochemical and thermal phases of chlorophyll a fluorescence. Photosynthetica 37:163182

Schansker G, Toth SZ, Strasser RJ (2006) Dark-recovery of the Chl a fluorescence transient (OJIP) after light adaptation: the $q(T)$ component of non-photochemical quenching is related to an activated photosystem I acceptor side. Biochim Biophys Acta 1757:787-797

Schilstra MJ, Rappaport F, Nugent JHA, Barnett CJ, Klug DR (1998) Proton/hydrogen transfer affects the S-state-dependent microsecond phases of $\mathrm{P} 680^{(+)}$reduction during water splitting. Biochemistry 37:3974-3981

Schreiber U (2002) Assessment of maximal fluorescence yield: donor-side dependent quenching and QB-quenching. In: van Kooten O, Snel J (eds) Plant spectrofluorometry: applications and basic research. Rozenberg Publ, Amsterdam, pp 23-47

Schreiber U (2004) Pulse-amplitude modulation (PAM) fluorometry and saturation pulse method: an overview. In: Papageorgiou G, Govindjee (eds) Advances in photosynthesis and respiration, vol. 19, Chlorophyll a fluorescence a signature of photosynthesis. Springer, Dordrecht, The Netherlands, pp 279-319

Schreiber U, Neubauer C (1987) The polyphasic rise of chlorophyll fluorescence upon onset of strong continuous illumination: II. Partial control by the photosystem II donor side and possible ways of interpretation. Z Naturforsch 42c:1255-1264

Shinkarev V (1996) Binary oscillations in the Kok model of oxygen evolution in oxygenic photosynthesis. Photosynth Res 48:411417

Shinkarev V (2004) Photosystem II: oxygen evolution and chlorophyll a fluorescence induced by multiple flashes. In: Papageorgiou G, Govindjee (eds) Advances in photosynthesis and respiration, vol. 19, Chlorophyll a fluorescence a signature of photosynthesis. Springer, Dordrecht, The Netherlands, pp 197229

Steffen R, Christen G, Renger G (2001) Time-resolved monitoring of flash-induced changes of fluorescence quantum yield and decay of delayed light emission in oxygen-evolving photosynthetic organisms. Biochemistry 40:173-180

Stirbet A, Govindjee, Strasser BJ, Strasser RJ (1998) Chlorophyll a fluorescence induction in higher plants: modelling and numerical simulation. J Theor Biol 193:131-151

Strasser RJ, Srivastava A, Govindjee (1995) Polyphasic chlorophyll a fluorescence transient in plants and cyanobacteria. Photochem Photobiol 61:32-42

Toth S, Schansker G, Strasser RJ (2005) In intact leaves, the maximum fluorescence level $(\mathrm{Fm})$ is independent of the redox state of the plastoquinone pool: a DCMU-inhibition study. Biochim Biophys Acta 1708:275-282
Urban O, Trtilek M, Field T, Nedbal L (1999) Single-turnover flashes to saturate the $\mathrm{q}_{\mathrm{a}}$ reduction in a leaf were generated by the lightemitting diodes from a double modulation kinetic chlorophyll fluorometer. Photosynthetica 37:201-207

van Gorkom HJ (1996) Electroluminescence. Photosynth Res 48:107_ 116

Vasilev S, Bruce D (1998) Nonphotochemical quenching of excitation energy in photosystem II. A picosecond time-resolved study of the low yield of chlorophyll a fluorescence induced by singleturnover flash in isolated spinach thylakoids. Biochemistry 37:11046-11054

Vermaas WFJ, Renger G, Dohnt G (1984) The reduction of the oxygen-evolving system in chloroplasts by thylakoid components. Biochim Biophys Acta 764:194-202

Vernotte C, Etienne AL, Briantais JM (1979) Quenching of the system II chlorophyll fluorescence by the plastoquinone pool. Biochim Biophys Acta 545:519-527

Vos MH, van Gorkom HJ, van Leeuwen PJ (1991) An electroluminescence study of stabilization reactions in the oxygenevolving complex of Photosystem II. Biochim Biophys Acta 1056:27-39

Vredenberg WJ (2000) A three-state model for energy trapping and chlorophyll fluorescence in photosystem II incorporating radical pair recombination. Biophys J 79:25-38

Vredenberg WJ (2004) System analysis and photo-electrochemical control of chlorophyll fluorescence in terms of trapping models of photosystem II: a challenging view. In: Papageorgiou G, Govindjee (eds) Advances in photosynthesis and respiration, vol. 19, Chlorophyll a fluorescence a signature of photosynthesis. Springer, Dordrecht, The Netherlands, pp 133-172

Vredenberg WJ, Rodrigues GC, van Rensen JJS (2001) A quantitative analysis of the chlorophyll fluorescence induction in terms of electron transfer rates at donor and acceptor sides of photosystem II. In: PS2001 Proceedings: 12th International congress on photosynthesis. CSIRO Publishing, Melbourne, CD-ROM, S3041

Vredenberg WJ, Kasalicky V, Bina D, Prasil O (2004) System analysis of chlorophyll fluorescence kinetics in chloroplasts: submaximal yield in single turnover excitations and electrical interactions. In: van der Est A, Bruce D (eds) PS2004 proceedings: 13th International congress on photosynthesis: from fundamental aspects to global aspects, vol I. Allen Press, Montreal, Canada, pp 190-192

Vredenberg WJ, van Rensen JJS, Rodrigues GC (2005) On the submaximal yield and photo-electric stimulation of chlorophyll $a$ fluorescence in single turnover excitations in plant cells. Bioelectrochemistry 68:81-88

Vredenberg WJ, Kasalicky V, Durchan M, Prasil O (2006) The chlorophyll a fluorescence induction pattern in chloroplasts upon repetitive single turnover excitations: accumulation and function of $\mathrm{Q}_{\mathrm{B}}$-nonreducing centers. Biochim Biophys Acta 1757:173181

Yaakoubd B, Andersen R, Desjardins Y, Samson G (2002) Contributions of the free oxidized and $\mathrm{Q}_{\mathrm{B}}^{-}$bound plastoquinone molecules to the thermal phase of chlorophyll-a fluorescence. Photosynth Res 74:251-257

Zhu X-G, Govindjee, Baker NR, deSturler E, Ort DR, Long SP (2005) Chlorophyll $a$ fluorescence induction kinetics in leaves predicted from a model describing each discrete step of excitation energy and electron transfer associated with Photosystem II. Planta 23:114-133 